\title{
VALUE ASSESSMENT AND IMPLEMENTATION TRADEOFFS FOR PRODUCTION HEAVY TRUCK ACTIVE NOISE CONTROL
}

\author{
Daniel J. Maguire \\ Active Systems \\ Cooper Advanced Technologies \\ Cooper Standard Automotive \\ Golden, CO
}

\begin{abstract}
Summary: Despite a long history, the implementation tradeoffs and value assessment criteria of active noise control (ANC) are not well established. With commercial heavy truck adaptive ANC controllers now available, there is a need for an understanding of the unique performance-to-cost ratio characteristics of these systems. Also, due to the specific spectral region that these systems are applicable to, studies of the physiological effects of broadband noise poorly represent the positive impact of ANC. This paper presents a collection of implementation tradeoff metrics unique to active noise control systems. In addition, evaluation issues and research are identified that will help to validate ANC benefits that are accepted, but insufficiently supported.
\end{abstract}

\section{INTRODUCTION}

Active noise control (ANC), which is the process of adding energy to a system to affect the destructive interference of unwanted sound, was the subject of a American patent granted to Paul Lueg in 1936 (Tokhi, 1992; Lueg, 1936). Lueg's depiction of active noise control took the form of a fixed closed-loop control system, or a controller whose parameters remain unchanged in the presence of changing conditions. Fixed controllers achieve satisfactory performance with stability over all conditions, so are inevitably a compromise of performance versus condition (Clarke, 1988).

An adaptive controller is defined as a controller with adjustable parameters and a means for adjusting those parameters (Astrom, 1995). Adaptive controllers use a measure of their performance to continually adjust their control behavior based on current performance to improve their performance for the next iteration, eventually approximating some optimal controller (Haykin, 1996). In this way, an adaptive control can react robustly to disturbance and system variance and still achieve some level of optimal control at each operating condition (Widrow, 1985).

Recent signal processing hardware cost reductions driven by high volume telecommunications applications have finally made economical commercial adaptive ANC systems available. For safety and comfort reasons, one obvious potential application area is the adaptive active noise control of combustion related acoustic energy at the driver and sleeper head areas in long haul truck cabs.

For an advanced technology to become a commodity, the costs, benefits, and design tradeoffs must be able to be identified and quantified. Despite the almost 70 year onset of commercial 
ANC technology, objective and subjective performance measures to quantify the value of adaptive active noise control are still lacking. In this paper, I will first describe the basic motivation of adaptive active noise control in heavy truck applications based on actual adaptive ANC implementations in commercial class 8 diesel trucks. I will then describe cost-performance tradeoffs inherent to adaptive active noise control and metrics representing these tradeoffs. Finally, some evaluation issues, including the need for investigations of noise-induced physiology specific to the application of adaptive active noise control that are crucial to establishing its value, will be described.

\section{THE APPLICATION OF ADAPTIVE ANC TO HEAVY TRUCKS}

Active control adds energy to a system. This can be contrasted to passive control approaches that dissipate energy. Dissipative noise control approaches, such as acoustic mats, are particularly cost- and weight-effective at frequencies greater that $600 \mathrm{~Hz}$. As frequencies decrease below $600 \mathrm{~Hz}$, the density/weight of passive sound absorption materials makes their application less practical.

ANC is truly a complimentary technology to passive noise control with respect to disturbance frequency. It is a matter of algebra to show that destructive noise interference in the near field of a secondary source creates a useful interference zone, or "quiet zone" with a radius, $\Delta r_{\varepsilon}=\lambda \sqrt{ } \varepsilon \pi$, where $\lambda$ is the wavelength of interest and $\varepsilon$ is the average squared pressure reduction ratio (Nelson, 1992). As a quiet zone smaller than a human head is ineffectual, this places the practical high frequency limit of ANC in the $200-300 \mathrm{~Hz}$ range. The low frequency limit of active noise control is limited by speaker size and back volume, amplifier capacity, and system memory limitations. For the Cooper ENVIsys ${ }^{\mathrm{TM}}$ production heavy truck active noise controller, $75 \mathrm{~Hz}$ is a typical setting for a low frequency bound, although truck cab booms below $50 \mathrm{~Hz}$ have been addressed.

Figure 1 shows the off/on spectra for a class 8 diesel truck with adaptive Cooper ANC applied to the driver and passenger seats while in a manufacturer specified cruise condition. The data shown was recorded by a microphone located at the driver ear area. It is readily seen from Figure 1 seen that the noise controller is able to reduce the low frequency tonal disturbance energy down to that of the random noise floor. This implementation resulted in $7 \mathrm{~dB}(\mathrm{~A})$ of measured reduction at the ear.

Figure 2 shows the time-domain broadband $(<5 \mathrm{KHz})$ microphone signal off/on transition for a Class 8 diesel truck with a Cooper production adaptive ANC applied to the sleeper head area. The vehicle was in a high idle condition typical of a refrigerated load. These data were acquired at a heavy truck manufacturers facility. The combination of idle RPM, sleeper cab geometry, and exhaust arrangement yielded an excited mode that was attenuated. The ANC implementation shown in Figure 2 resulted in a 9dBA reduction in SPL at the sleeper head area.

For the purposes of the rate of reduction of driver fatigue and the increase in sleeper berth sleep quality, these examples of actual production adaptive active noise control are presented as compelling examples of the positive benefit of heavy truck ANC. 


\section{COST-PERFORMANCE OR SYSTEM TRADEOFFS}

Ignoring the particular algorithm of an adaptive active noise controller, some basic adaptive ANC cost-performance tradeoffs exist for heavy truck implementations.

\section{Acoustic or Computational Delays vs. Dynamic Performance and/or Stability}

Acoustic delays are the result of the propagation of the controller output noise to the error sensor or microphone. This delay could be caused by physical distance or reflection. There is a desire to increase the acoustic delay of an active noise control system for aesthetic reasons. Rather than give up storage space behind the driver and/or passenger, placing the speaker in a less useful and more remote space seems desirable. Just as with the case of fixed feedback control, this has a direct effect on either dynamic performance (tracking), algorithmic stability or both.

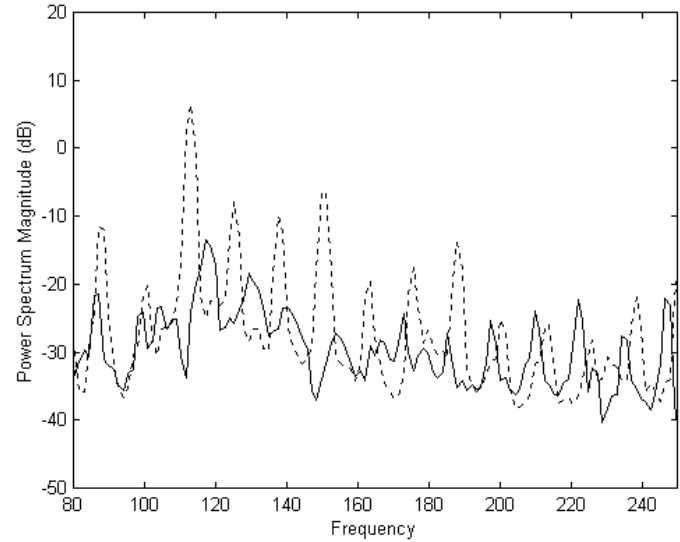

Figure 1 - Driver ear off (dotted) and on spectrum (solid) for a Class 8 diesel sleeper cab with ANC. The unit was being run at a manufacturer specified cruise RPM.

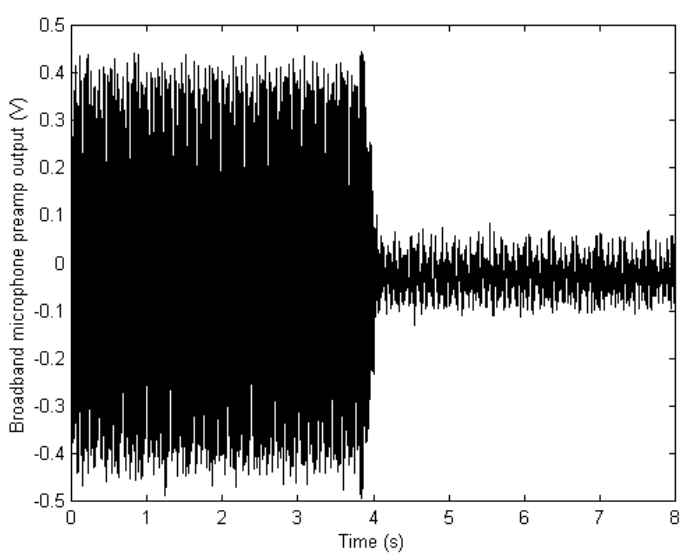

Figure 2 - Broadband $(0-5 \mathrm{KHz})$ timedomain recording at the sleeper's ear in a Class 8 diesel sleeper cab with ANC. The unit was being run at high idle.

This tradeoff requires a quantitative/qualitative measure of dynamic performance degradation or loss of stability margin vs. the aesthetic impact of and useful space consumed by the speaker.

Computational delays are the result of using slower processors or certain signal processing components or algorithms. An increase in computational delay typically results in a reduction of cost with a corresponding decrease in dynamic performance and/or algorithmic stability.

At the algorithmic level, the stability tradeoff can be quantified in terms of a unit of stability margin per inch, millisecond, or degree. For tracking performance, a lower bound of minimum $\mathrm{dB}$ attenuation per harmonic per RPM per second can be defined. Using the vehicle's maximum $\triangle \mathrm{RPM}$, a maximum delay can then be estimated.

\section{Steady-state Performance vs. Dynamic Stability}

Adaptive algorithms, in general, trade reluctance to change during fixed conditions with aggressiveness of change during dynamic conditions, to some degree. This tradeoff requires a quantitative measure of steady state performance degradation with respect to disturbance rate of 
change. Choosing a lower bound for both the attenuation during steady state conditions and the attenuation degradation during dynamic disturbance changes in $\mathrm{dB}$ attenuation per harmonic per RPM per second would sufficiently define the desired emphasis of the controller.

\section{Ear-to-error Microphone Distance vs. Perceived Benefit}

Without algorithmic extensions, the quiet zone of the controllers is centered about the error sensor. Independent of acoustic delay, the degree with which the driver or passenger's head is eccentric to the quiet zone will cause a loss in perceived effect. The ideal sensor location is often at the driver's ear. As with the speaker placement, a remote sensor location would be preferred by most manufacturers for aesthetic reasons. Arbitrating this tradeoff requires a quantitative/quantitative measure of steady state or dynamic performance loss vs. frequency vs. microphone aesthetic impact.

\section{Number of Control Zones vs. Cost}

Due to the basic need to consider cross effects in multi-input/multi-output systems, the computational complexity of an adaptive algorithm generally increases with the square of the number of controlled locations. This means that a single system that controls the driver, passenger, and sleeper locations will require roughly 9x the computations of a single seat system. In general, the additional sites will improve the attenuation achieved with the system. Consequently, this tradeoff requires a compromise of control sites vs. increased performance vs. system cost.

\section{EVALUATION ISSUES}

A performance component exists in all metrics described, and yet system performance in $\mathrm{dB}(\mathrm{A})$ is an abstract measure of the true value of any noise reduction. The following issues confuse the heavy truck manufacturers assessment of the true value of ANC to the end customer.

\section{Varying Sensitivities to Low Frequency Noise}

Studies have established that individuals have differing sensitivities to low frequency noise (LFN) (Waye, 2000). In addition, establishing a value criterion for ANC is complicated by the fact that some truckers that have been subjected to LFN throughout their careers have lost low frequency sensitivity. They would perceive no value in ANC, or any other technology reducing LFN.

\section{ANC Effect Dependent Upon Operation Condition and Vehicle Design}

The goal of a non-headphone based active noise controller is to reduce unwanted noise components to the level of the noise floor. If a vehicle is driven under conditions where combustion-related components are not elicited, or if the vehicle is poorly treated passively, the ANC effect will be masked. An identical active effect can be perceived as substantial or imperceptible, based entirely on noise existing outside the ANC spectral range, and independent of the control action. 


\section{dB(A) a Poor Measure of the Actively Controlled Spectrum}

The A-weighted scale, on which most federal regulation limits are based, penalizes the low frequency spectral region as imperceptible. The trucking industry would be better able to measure the benefit of $A N C$ by adopting the simultaneous use of $\mathrm{dB}(\mathrm{A})$ and $\mathrm{dB}(\mathrm{C})$. In this way, low and high frequency content (and the impact of ANC) would be properly measured (Berglund, 1995).

\section{Limited Research Exists Specifically Addressing Low Frequency Human Physiological Effect}

Truck driver fatigue and fatigue detection has been the subject of many federal research projects (Knipling, 2000). Broadband noise impact on fatigue and sleep quality has also been investigated and some relationships have been documented (Hanowski, 1998; Neale, 2000). Still, the effect of low frequency noise content on a driver's fatigue, rate of fatigue, and annoyance has not been a popular topic of study (Powell, 1995; Isaacs, 2000). Studies have shown a loss of performance on simple tests and physiological indications of increased stress in the presence of LFN as quiet as $40 \mathrm{~dB}$ LAeq (Waye, 2000; Bengtsson, 2000). LFN has been linked to increased heart infarct, chronic insomnia, and depression (Mirowska, 2000). LFN also masks speech more than high frequency noise, complicating communication and increasing frustration (Songer, 1992).

High noise levels in truck cabs impede communication; mask warning signals; and damage driver hearing. It has even been recommended that heavy trucks be fitted with technology to reduce noise levels, not as an option on the best trucks, but on all trucks as standard equipment (Lee, 1998)

\section{CONCLUSION}

Despite almost 70 years of history, the integration of now commercially available ANC systems will be difficult without a basis on which to establish value. To combat the lack of a value criterion, this paper intended to present basic performance-to-cost ratio tradeoff metrics unique to active noise control. In all cases, system performance is one of two or more dimensions of the metric.

In addition to implementation tradeoffs, ANC evaluation issues were discussed. These include vehicle variations, operator variations, the inappropriateness of the A-weighted measurement scale, and the lack of research into the specific physiological effect of low frequency noise content while driving. It is hoped that through increased investigation into ANC-specific noise reduction benefits, that the application of ANC to the heavy truck market will be hastened.

\section{REFERENCES}

Bengtsson, J., Waye, K.P., Kjellberg, A., and Benton, S. (2000). Does low frequency noise during work induce stress? Internoise 2000, Nice FR, Aug. 27-30.

Clarke, D.W. (1988). Introduction to Self-tuning Control. Implementation of self-tuning controllers. Peter Peregrinus Ltd., London. 
Hanowski, R.J., Weirwille, W.W., Gellatly, A.W., Dingus, T.A., Knipling, R.R., and Carroll, R. (1998). Driver's perspective on fatigue in long/short haul trucking. SAE International Truck and Bus Exposition, Indianapolis, IN, Nov. 16-18.

Haykin, S. (1996). Adaptive Filter Theory, 3rd. Ed. Prentice-Hall, Inc. Upper Saddle River, NJ.

Isaacs, A. (2000) Noise induced hearing loss. http://wings.buffalo.edu/faculty/research/chd/NIHL

Knipling, R.R. (2000). Driver alertness and fatigue: Summary of completed research projects, 1995- 98. FMCSA Tech Brief, Office of Motor Carrier Research and Standards, Wash. D.C.

Lee, S.E. (1998). Role of driver hearing in commercial motor vehicle operation: An evaluation of the FHWA hearing requirement. Ph.D. Dissertation, Virginia Polytechnic and State University, Blacksburg, VA.

Lueg, P. (1936). Process of silencing sound oscillations. US Patent No. 2043416.

Mirowska, M. and Mroz, E. (2000). Effect of low frequency noise at low levels on human health in light of questionnaire investigation. Internoise 2000, Nice FR, Aug 27-30.

Neale, V.L., Robinson, G.S., Dingus, T.A, and Davis, R.E.L. (1998). Long haul driver's perspective on sleeper berth usage and fatigue in the trucking industry. SAE International Truck and Bus Exposition, Indianapolis, IN, Nov. 16-18.

Nelson, P.A., and Elliot, S.J. (1992), Active Control of Sound. University Press, Cambridge.

Powell, C.A. and Fields, J.M. (1995). Human response to aircraft noise. Aeroacoustics of flight vehicles: Theory and practice - Volume 2: Noise control. Ed. Hubbard, H.H. ASA, NY.

Songer, T.J., Lapprte, R.E., Palmer, C.V., Law, L.B., Talbot, E., Gibson, J.S., and Austin, A. (1992). Hearing disorders and commercial vehicle drivers. FHWA-MC-93-004.

Tokhi, M.O. and Leitch, R.R. (1992). Active Noise Control. Oxford Science Publications, Oxford.

Waye, K.P., Rylandere, R., Bengtsson, J., Clow, A., Hucklebridge, F., and Evans, P. (2000). Does low frequency noise during work induce stress? Internoise 2000, Nice FR, Aug 27-30.

Widrow, B., and Stearns, S.D. (1985). Adaptive Signal Processing. Prentice-Hall, Inc., Englewood Cliffs, NJ. 